\title{
Transatlantica
}

Revue d'études américaines. American Studies Journal

\section{« La machine dans la littérature et les arts visuels du monde anglophone »}

Journée d'études internationale OVALE, Sorbonne-Université

28 mai 2019

\section{Olivier Hercend}

\section{OpenEdition}

\section{Journals}

Electronic version

URL: https://journals.openedition.org/transatlantica/11509

DOI: 10.4000/transatlantica. 11509

ISSN: $1765-2766$

Publisher

Association française d'Etudes Américaines (AFEA)

Electronic reference

Olivier Hercend, "«La machine dans la littérature et les arts visuels du monde anglophone »",

Transatlantica [Online], 1 | 2018, Online since 07 October 2019, connection on 01 February 2023. URL: http://journals.openedition.org/transatlantica/11509; DOl: https://doi.org/10.4000/transatlantica. 11509

This text was automatically generated on 1 February 2023

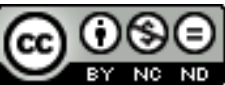

Creative Commons - Attribution-NonCommercial-NoDerivatives 4.0 International - CC BY-NC-ND 4.0 https://creativecommons.org/licenses/by-nc-nd/4.0/ 


\section{« La machine dans la littérature et les arts visuels du monde anglophone »}

Journée d'études internationale OVALE, Sorbonne-Université

28 mai 2019

Olivier Hercend

1 Cette journée d'études internationale, organisée par les doctorantes Diane Drouin, Anouk Bottero et Omayyah Al-Shabab, avec le soutien de l'équipe de recherche VALE (EA 4085) et de l'ED IV, à Sorbonne-Université, constituait l'aboutissement du séminaire doctoral du laboratoire OVALE, qui portait cette année sur le thème de «La machine dans la littérature et les arts visuels du monde anglophone». Fidèles à l'esprit d'ouverture de cette équipe, les contributions alliaient réflexions sur l'art, la science et la société, dans une perspective transdisciplinaire et transatlantique. Loin de la vision étroite de la machine-objet, l'approche englobait toutes les facettes du mécanisme : à la fois comme concept philosophique, dans son rapport d'opposition et de complémentarité avec l'organique et avec l'humain, comme métaphore politique et sociale, et dans son historicité, consubstantielle avec celle des arts et techniques. Comme le rappelait en ouverture Line Cottegnies (Professeure à Sorbonne-Université, directrice-adjointe et représentante pour l'occasion de l'équipe VALE), la pensée de la machine est toujours tributaire d'une vision du monde, de ses rouages, mais également des potentialités créatrices qui l'habitent. En cela, le travail artistique et intellectuel de l'invitée, J.R. Carpenter, représentait une parfaite entrée en matière.

\section{J.R Carpenter : « Mapping Machines : Rewriting the World »}

2 Artiste reconnue et docteure de la University of Arts, London, J.R. Carpenter s'est présentée dès l'abord comme engagée dans une œuvre de destabilisation des ordres et des hiérarchies. Canadienne en Angleterre, migrante et descendante de migrants, elle cherche à tracer d'autres lignes que les lignes de force, coloniales, capitalistes ou consuméristes, qui strient les cartes du XXI ${ }^{\text {ème }}$ siècle. Parlant de son amitié avec une 
« interlocutrice absente ", l'artiste Mary Patterson, elle a évoqué leurs dialogues, et ses tentatives paradoxales pour répondre aux questions sans réponse (" unanswerable ) qui étaient nées de ces échanges. Or, ce « sans réponse » défie les tentatives récentes de contrôle de l'espace, qui assignent un code et une série d'informations à chaque point du globe. C'est face à ce catéchisme sans fin de Google Maps qu'elle a choisi d'affirmer l'irréductible épaisseur du concept de «lieu », de «foyer », de « chez-soi » - autant de forces échappant à la traçabilité des méta-données. Dans «Les huit quartiers du Sommeil », elle surimpose à une image satellite les récits, réels ou fictifs, d'habitants de Montréal, confrontant l'espace vécu à la vue verticale et plate de l'image satellite. Sa réflexion, héritière de celles de Richter et Benjamin, et après eux Adorno et Derrida, vise ainsi une restructuration de l'espace, qui passe par une pensée renouvelée du temps. Utilisant depuis les années 1990 les potentialités de l'internet, les «sites-web » et leurs architectures non-linéaires, Carpenter resitue les tentatives récentes d'appropriation des lieux - surveillance satellite, méta-données, gentrification - dans une histoire plus large. D'archive en archive, elle superpose les strates de cartographies, les efforts de modélisation successifs, en détectant les projections, les entreprises de contrôle, mais aussi les erreurs et les rêves humains qui s'y lisent. Son travail récent, "Le plaisir de la côte ", réalisé auprès de l'université Paris 8 et des Archives Nationales, prolonge cette veine : elle explore les traces d'une exploration, et fait remonter à la surface le processus d'appropriation par l'homme du territoire. Par ces biais, celle qui affirme être d'abord "collage-artist » brise l'illusion d'un progrès simple des techniques, au profit d'une insistance temporelle, une coexistence des strates, qui est l'histoire secrète des machines cartographiques et de leurs emplois.

\section{Panel 1 : Machines théâtrales.}

3 Le premier panel de la journée a vu s'entrecroiser deux aspects complémentaires du théâtre, la machinerie sociale de la performance venant faire contrepoint à l'abstraction des formes. Mylène Maignant, doctorante à l'École normale supérieure de Paris, explorait dans son intervention « Mathematics and Computer Science on Samuel Beckett's Stage » les potentialités d'outils numériques et de modélisation, pour rendre compte de la mécanique scénique dans les pièces Fin de Partie et Quad de Samuel Beckett. Comme elle l'a précisé en réponse à une question, sa recherche ne visait pas à rendre compte d'une intention auctoriale du dramaturge, mais plutôt, par le biais d'outils nouveaux, à proposer un autre cadre de lecture des didascalies de ces pièces. Stylisant l'espace scénique, changeant les acteurs en carrés de couleurs qui traçaient à vitesse constante des figures géométriques, elle affirmait le parallèle entre l'abstraction croissante du théâtre de Beckett et les découvertes de la cybernétique après la Seconde Guerre mondiale. Reste ce "bruit ", mentionné à la fin de sa présentation : ce qu'elle ne pouvait modéliser, et qui semblait constituer la face obscure de sa propre pensée autant que de ses outils. C'est justement sur les conditions matérielles de la représentation, et l'ingénierie scénique, qu'Elise Rale, doctorante à Sorbonne-Université, se penchait dans "Les machines de Broadway ». Analysant la tendance récente à une surenchère d'effets mécaniques dans le théatre contemporain de Broadway, elle proposait un retour en arrière sur le théâtre américain, ses conditions de production, son rapport à l'architecture et aux arts décoratifs, et en dernières instance son modèle économique. Ces réflexions menaient à une remise en question de l'« effet de réel » dans ce théâtre, à travers les notions d'« hyperréalité » et de « real deal » développées par Umberto Eco : 
plutôt qu'un quelconque naturalisme, le théâtre américain chercherait avant tout à susciter une forme de "plaisir enfantin » et "magique ", réalisant l'utopie d'une scène changée en monde. Cette volonté d'oublier les conditions de représentation, cet idéal du mécanisme qui s'efface lui-même pour « faire plus vrai », est une tendance dont Rale proposait de voir les effets au-delà des seules machines-objet, dans le jeu même des acteurs, leur rapport à leur performance et jusqu'à leur propre corps.

\section{Panel 2 : Les machines de la modernité : de l'inquiétude à la fascination} esthétique

4 Trois présentations successives retraçaient ici, à travers plus d'un siècle et de l'Amérique à Vladivostock, les élans mécaniques de la modernité. Dans son intervention intitulée "'Smoothing the ravell'd fleece': spinning machine between poetry and politics", Tatiana Smoliarova, professeure associée à l'université de Toronto, repartait des racines de la révolution industrielle, pour sonder l'impact langagier et poétique de la mécanisation. Elle se concentrait surtout sur le poème « The Botanic Garden » d'Erasmus Darwin, repérant ce que cette œuvre didactique - qui voulait être pour Linné ce que le poème de Pope "An Essay of Man» avait été pour Newton - avait repris au monde des machines, comme "stock de métaphores » pour décrire la nature. Ce mélange de l'organique et des inventions nouvelles révélait en dernière instance à quel point l'élément langagier se nourrissait de matérialité, et à quel point les instruments de l'homme influaient sur sa perception du monde. L'impact mondial de la révolution industrielle fut ainsi abordé, à la fin de la présentation, à travers la réception en Russie des machines à filer importée du Royaume-Uni, et leur intégration comme métaphores pour parler des conditions de vie dans le pays. Traversant l'atlantique, Pauline Choay-Lescar (Sorbonne-Université) présenta dans "Whitman et les temps modernes » les rapports complexes du poète américain avec le monde des machines. De l'épopée de la conquête du continent à celle, verticale, de l'érection des grattes-ciel, ses textes explorent les potentialités de la technique. A la fois chantre de la technologie, du lien entre l'organique et le mécanique ( I sing the body electric»), et fasciné par les avancées "spirituelles» des transports et des communications, qui recomposaient l'espace et le temps, Whitman n'en remarquait pas moins les facettes plus inquiétantes de la mécanisation. Outils du travailleur et du créateur, les machines semblent à mesure que sa poésie mûrit prendre une autonomie de plus en plus grande, que Choay-Lescar liait avec la disparition progressive du «je » poétique - et l'autonomisation croissante de la machine textuelle, du corps même du poème. Enfin, Tamar Kharatishvili, doctorante à Northwestern University, abordait le $\mathrm{XX}^{\mathrm{ème}}$ siècle dans « Expatriate Modernisms: Machine Aesthetics in Pound, Cendrars and Delaunay-Terk ». Par une lecture des théories d' Ezra Pound et de T.E. Hulme à la lumière de «La prose du transsibérien » de Cendrars, illustré par Sonia Delaunay-Terk, celle-ci opposait la dynamique d'appropriation et de condensation triomphale des premiers dans les années 1910, à l'impression de perte de repères et à l'esthétique hybride des seconds. Mettant en parallèle le travail de Delaunay-Terk sur le poème et son intérêt pour les halos des lumières électriques, tels qu'elle les peint dans «Prismes électriques ", elle proposait une réévaluation, plus ambivalente et plus singulière, du poème, loin de l'exaltation futuriste pour l'ère des machines, et empreint déjà du « doute » qui s'emparerait du modernisme d'après-guerre. 


\section{Panel 3 :Perspectives américaines contemporaines sur la machine}

5 Le dernier panel se focalisait sur le monde contemporain, à mi-chemin entre l'idéal d'une origine perdue et la perspective de l'Apocalypse. Dans «L'Âge de cristal (Michael Anderson, 1976), ou la ville-machine et la contre-utopie pastorale », Mehdi Achouche, maître de conférence à l'Université Jean Moulin - Lyon 3, abordait le rapport à l'utopie mécanique dans le cinéma hollywoodien des années 1970. L'allégorie présentée dans le film (Logan's Run en anglais) reliait l'artificialité des machines et des décors (le film était tourné dans un centre commercial dernier cri) à la décadence de la société. Celle-ci s'était engouffrée dans une version caricaturale du mode de vie consumériste et de l'hédonisme prôné par le mouvement hippie. Il fallait alors un voyage initiatique au couple des héros, vers la clairière édénique qu'était devenue Washington D.C., pour réapprendre les vraies bases de l'éthique américaine : le travail pour obtenir les fruits de la nature, le respect pour les hommes vieux - Abraham Lincoln, et ce sage vieillard qui garde la constitution dans les locaux du Sénat - et les vertus du couple monogame et du mariage. Cette vision conservatrice de l'anticipation, qui signalait selon Achouche le lien très étroit entre la dystopie américaine et les racines utopiques de la fondation du pays, contrastait fortement avec les visions foisonnantes et diverses que proposait Héloïse Thomas, doctorante à l'université Bordeaux Montaigne, dans « Machines in the Anthropocene ». Rappelant la définition géologique puis littéraire de l'« anthropocène ", comme scission entre la nature et son exploitation mécanique au sein des systèmes institutionnels du capitalisme et du patriarcat, celle-ci se proposait de définir plus avant la place de la machine dans cette opposition. En effet, si la machine n'est ni purement ennemie ni instrument $d u$ salut dans la fiction d'anticipation contemporaine, la question de son usage devient le point de focalisation central. En proposant de revoir l'interaction avec le monde et avec soi-même, d'ouvrir les mécanismes sociaux comme matériels à la créativité, les fictions d'anticipation de la littérature «queer » explorent les hybridations auxquels les interactions entre humain et machines donnent lieu. Prenant l'exemple du recueil Full-metal Indigiqueer de Joshua Whitehead et du roman Blackfish City de Sam J. Miller, Thomas montrait l'irruption du programme dans les mécanismes du langage, et de la machine dans le champ de l'intrigue. Loin d'être révélation finale, l'Apocalypse devenait, au prisme de cette lumière " queer », un champ d'exploration privilégié de l'humain.

\section{Conclusion}

6 En effet, à travers les siècles, de la «mule-Jenny » aux perversités futuristes de la science-fiction, la machine s'est présentée à nous lors de cette journée d'études avant tout comme un médiateur symbolique de l'homme au monde et de l'homme à l'homme. Elle est outil, mais aussi signe, sur lequel se trouvent projetés les espoirs et les angoisses, les idéologies et les résistances des individus ainsi que des sociétés dans leur ensemble. Plutôt que de modéliser un champ dans son développement mécanique, il a semblé que nous poursuivions une multitude de pistes, de lignes de fuite vers le passé ou l'avenir rêvé, qui filent le long des filins d'acier, des poutres, des escalators, de ces insondables traînées de fumée qui s'échappent de la locomotive dans le tableau de Turner « Rain, Steam and Speed ». Et toutes, à l'analyse, partaient des mains, des yeux et des esprits des hommes, et se trouvaient inscrites dans le langage, reflet « spirituel » et intersubjectif du monde de la technique. 
INDEX

Subjects: Actualité de la recherche

\section{AUTHOR}

OLIVIER HERCEND

doctorant, Sorbonne-Université 